\title{
The interaction of socioeconomic status with place of death: a qualitative analysis of physician experiences
}

\author{
Joshua Wales*, Allison M. Kurahashi and Amna Husain
}

\begin{abstract}
Background: Home is a preferred place of death for many people; however, access to a home death may not be equitable. The impact of socioeconomic status on one's ability to die at home has been documented, yet there remains little literature exploring mechanisms that contribute to this disparity. By exploring the experiences and insights of physicians who provide end-of-life care in the home, this study aims to identify the factors perceived to influence patients' likelihood of home death and describe the mechanisms by which they interact with socioeconomic status.

Methods: In this exploratory qualitative study, we conducted interviews with 9 physicians who provide homebased care at a specialized palliative care centre. Participants were asked about their experiences caring for patients at the end of life, focusing on factors believed to impact likelihood of home death with an emphasis on socioeconomic status, and opportunities for intervention. We relied on participants' perceptions of SES, rather than objective measures. We used an inductive content analysis to identify and describe factors that physicians perceive to influence a patient's likelihood of dying at home.

Results: Factors identified by physicians were organized into three categories: patient characteristics, physical environment and support network. Patient preference for home death was seen as a necessary factor. If this was established, participants suggested that having a strong support network to supplement professional care was critical to achieving home death. Finally, safe and sustainable housing were also felt to improve likelihood of home death. Higher SES was perceived to increase the likelihood of a desired home death by affording access to more resources within each of the categories. This included better health and health care understanding, a higher capacity for advocacy, a more stable home environment, and more caregiver support.
\end{abstract}

Conclusions: SES was not perceived to be an isolated factor impacting likelihood of home death, but rather a means to address shortfalls in the three identified categories. Identifying the factors that influence ability is the first step in ensuring home death is accessible to all patients who desire it, regardless of socioeconomic status.

Keywords: Palliative care, Social class, House calls, Socioeconomic factors, Healthcare disparities, Place of death

\footnotetext{
* Correspondence: Joshua.wales@sinaihealthsystem.ca

The Temmy Latner Centre for Palliative Care, Sinai Health System, 60 Murray

Street, 4th Floor, Box 13, Toronto, ON M5T 3L9, Canada
}

(c) The Author(s). 2018 Open Access This article is distributed under the terms of the Creative Commons Attribution 4.0 International License (http://creativecommons.org/licenses/by/4.0/), which permits unrestricted use, distribution, and reproduction in any medium, provided you give appropriate credit to the original author(s) and the source, provide a link to the Creative Commons license, and indicate if changes were made. The Creative Commons Public Domain Dedication waiver (http://creativecommons.org/publicdomain/zero/1.0/) applies to the data made available in this article, unless otherwise stated. 


\section{Background}

Home has emerged as the preferred place of death for most patients [1-3] and as a cost-effective alternative to institutionalized death [4]. Studies have shown that access to home death is influenced by a number of personal factors, including age [5, 6], sex [5], education [7], disease type [8,9], marital status or cohabitation $[6,10-12]$, preferred place of death [13], ethnicity $[8,10]$, informal support networks [6, 14], and cultural affiliations [8]. Access to care in the home has also been associated with home death. Previous studies investigating the availability and utilization of home care and home visits found that patients were more likely to die at home or out of acute care if they lived in an area with more home hospice providers [10], received home care in the last 6 months of life [15], or received more nursing and personal support worker visits $[6,16,17]$.

Associations between socioeconomic status (SES) and place of death in the literature are inconsistent. Several studies found that rates of home death are lower for patients defined as lower SES or low income [10, 12, 17-24]. Conversely, other studies did not find a significant association between place of death and socioeconomic status based on perceived or actual income level [11, 25-29], deprivation score [30], or education level [31].

Previous studies that have identified SES-dependent differences in rates of home death have hypothesized factors to explain these disparities: patient or family capacity to provide adequate care at home [18, 22], level of access to care services [20, 22], personal preferences [22], care-related costs [18], control over monetary resources [23], and ability to secure local supports [20]. Few studies, however, have directly explored the mechanisms by which these determinants - including SES - result in disparities of home death rates. A more complete understanding of these socioeconomic determinants of home death may provide some insight into the inconsistent findings in the literature and help to address SES-related inequities that may exist in accessing home death.

This exploratory study aims to identify the key factors perceived to influence likelihood of home death and define the mechanisms by which they interact with SES. The findings presented here are based on the experiences of physicians who provide home-based palliative care in an urban Canadian context. In future research, we will use the factors identified in this study to refine the research question and capture the perspectives of patients, families and other healthcare providers. They may also highlight areas of opportunity for policy and practice development to mitigate potential disparities within a home-based palliative care context.

\section{Methods}

\section{Study design}

Working within a Qualitative Description framework [32], the investigators used inductive qualitative content analysis [33] to describe the factors that may influence the likelihood of home death and describe how these factors interact with patient socioeconomic status. Qualitative description studies aim to provide a comprehensive summary of a phenomenon that can be used as an entry point for further study [32]. Our data were informed by physician's perceptions of their patients' SES and did not employ empiric indices of patients' socioeconomic statuses. At the time of recruitment, the principal investigator (JW) was completing his residency and was known to participants as a colleague and student. $\mathrm{He}$ currently practices as a staff physician at the centre. The study coordinator (AK) has experience conducting research interviews but is not a clinician. She was known by all participants. The study protocol was approved by the local Research Ethics Board. The methods and findings are described according to the consolidated criteria for reporting qualitative research (COREQ) guidelines [34].

\section{Population}

We interviewed physicians whose clinical practice is focused on providing palliative care services to individuals at home. Physicians providing home-based palliative care may be well placed to comment on the determinants of home death. First, they can compare between their experiences and interactions with different patients and patient support networks, which occur in a home environment. Second, their experiences leading goals of care discussions can inform insights into the decision-making processes around place of death. Finally, they can root their perceptions about patient SES in their experiences discussing financial capacity with patients when planning care services.

In order to generate a deep understanding of the factors perceived within a home-based palliative care context, we recruited physicians from a single palliative care centre in Toronto, Ontario. The centre is one of the largest home palliative care programs in Canada, and offers 24/7 physician support to patients with life-limiting illness in their homes. Seventeen physicians dedicate an average of 13 full time equivalents (FTEs) to home care practice. Physicians have had a focused practice in palliative care at the centre ranging from 1.5 to 20 years. The area served by the palliative care centre has nearly equal distribution between income quartiles [16] but incudes neighbourhood clusters of both low- and high-income households [35]. The catchment area is divided into smaller zones, which are serviced by one or two physicians each. Because each zone has a distinct 
socioeconomic and cultural make-up, some physicians may service areas with a higher density of low-income households than other physicians.

In 2016, the centre recorded 12,551 encounters (physician calls or home visits) with patients. Both types of encounters are necessary to provide palliative care in patients' homes. On average, there were 80.5 encounters per physician FTE per month, and each patient experienced an average of 5.9 encounters.

\section{Sample size and recruitment}

The principal investigator sent an email invitation to participate in the study to all physicians practicing with the palliative care centre who provide home-visits. Interested physicians contacted the study coordinator to schedule an interview. Of the 17 physicians contacted, 9 participated in the study. Four physicians were not responsive, three had scheduling conflicts, and one declined as they felt their experiences would not contribute to understanding the research question. Participants were made aware of JW's role in the study and the safeguards that were used to preserve privacy during the consenting process.

\section{Data collection}

The semi-structured interview guide was created and refined by JW and AK through a process of evaluating each question's relevance to the overall study aim. The final interview guide (Appendix 1) consisted of a mix of open ended and quantitative questions. An interview preamble informed patients that the researchers were interested in the experiences of home-care palliative physicians and the factors they believe to influence a patient's ability to die at home. Participants were not given definitions for the terms "high" and "low" socioeconomic status, nor patient economic data. Earlier questions probed physicians' general experiences and factors believed to influence the likelihood of home death. Later questions targeted factors related to socioeconomic status, discrepancies resulting from SES, and strategies to address these discrepancies. Individual or telephone interviews were conducted during February 2016. AK conducted all interviews in order to protect participant confidentiality and reduce potential response bias that may have presented if the principal investigator had conducted the interviews.

\section{Data analysis}

All interviews were audio recorded, professionally transcribed verbatim and de-identified to maintain participant privacy. AK compared completed transcripts to audio recordings to verify accuracy. Based on AK's field notes, the researchers periodically met to discuss emerging concepts, noting ones that were new or redundant with earlier interviews. Based on these reviews, greater focus was placed on questions probing socioeconomic status and caregiver support levels after the first 6 interviews were completed. Three more interviews were conducted, but no new concepts emerged. Two reviewers (JW \& AK) independently open-coded the first three interview transcripts using NVivo version 10 software, generating detailed, non-hierarchical lists of codes. The reviewers then met to compare and refine their list of open codes (64 codes). At this time, duplicate codes were merged and the resulting 37 codes were defined and organized into five categories: Patient Factors, Caregiver Factors, System Factors, Socioeconomic Factors and Strategies to decrease disparity. The reviewers independently coded all nine interview transcripts according to this coding framework, applying all relevant codes to capture interactions between factors. The reviewers met again to compare their application of codes. Any discrepancies were resolved through discussion. Data within each category were reviewed and grouped into sub-categories [33], which represent key factors. The researchers used visual schemata to conceptualize the relationships between factors within and between categories. Factors were continually compared against the primary data to ensure accurate representation. This iterative process of categorization and comparison was continued until no new factors emerged. Because the consistency of concepts across interviews demonstrated the stability of the coding framework (i.e. no new codes were added), and because no new factors were emerging from ongoing data abstraction, the researchers agreed that saturation had been reached [33, 36-38], and that no additional interviews were required. If, following analysis, saturation had not been reached, efforts would have been made to schedule interviews with those physicians who previously had scheduling conflicts. Given JW's position at the palliative care centre, efforts were made to recognize his own personal clinical experiences, and how they informed the analysis of the data. Any conclusions drawn were discussed with the study coordinator and examined against the data to ensure they were not unduly coloured by biases derived from his clinical experience.

Counts and percentages were calculated for Yes/No questions. Median and range were calculated for the question asking about percentage of patient population felt to be of lower SES.

\section{Results}

Participant responses to quantitative questions are presented in Table 1. All participants indicated that they had cared for patients they would consider to be of lower SES. When describing their current practice, participants indicated that on average, approximately $30 \%$ 
Table 1 Participant quantitative responses

\begin{tabular}{llll}
\hline Participant & $\begin{array}{l}\text { Q5: Have you ever cared for patients } \\
\text { you would consider to be of lower } \\
\text { socioeconomic status? (Yes or No) }\end{array}$ & $\begin{array}{l}\text { Q6: What percentage of your current } \\
\text { patent population would you consider } \\
\text { to be of lower socioeconomic status? (\%) }\end{array}$ & $\begin{array}{l}\text { Q7: Do you feel that there is a difference in } \\
\text { a patient's ability to die at home depending } \\
\text { on if they are of lower or higher socioeconomic } \\
\text { status? (Yes or No) }\end{array}$ \\
\hline 1 & Yes & 30 & Yes \\
2 & Yes & 38 & Yes \\
3 & Yes & 33 & Yes \\
4 & Yes & $5-10$ & Yes \\
5 & Yes & 90 & Yes \\
6 & Yes & $10-20$ & No \\
7 & Yes & 90 & Yes \\
8 & Yes & 20 & Yes \\
9 & Yes & $20-25$ & Yes \\
\hline
\end{tabular}

of their patients (range 7.5-90\%) could be considered to be of lower socioeconomic status, reflecting the different areas served by each physician. All but one participant (89\%) indicated that they believed socioeconomic status had an impact on a patient's ability to die at home.

During the 20-40 min interviews, physicians described several factors that they perceived to impact ability to die at home. These factors were grouped into three categories: patient characteristics, physical environment, and support network (Table 2). While a higher perceived SES was not identified as a primary determinant of a successful home death, it was consistently noted to strengthen the other key categories that were identified. Below, we describe each factor, how they are perceived to interact with SES, and possible strategies to decrease disparities in accessing home death.

\section{Patient characteristics}

Two patient characteristics were believed to influence a patient's ability to die at home: their preferences about place of death, and their ability to navigate the health care system. These factors were informed by perceptions of a patient's background, culture and character traits as well as their physical, emotional or mental state that were felt to impact their likelihood of dying at home.

Patient preference was seen as a key determinant of place of death. Physicians reported that patient preference for home death was often determined by emotional factors, such as patient and caregiver discomfort, fear, or anxiety. These emotions were mainly centred around fear of coping poorly, perceived or actual burden on caregivers, and the potential lasting impact of home death on family members.

“There are some people for whom it's not a number of hours or amount of money issue, they just don't feel comfortable dying at home"... "When given the choice of palliative care unit or home, and somebody doesn't want to put their family through something, it doesn't matter if they can hire caregivers or not... because it's the emotional aspect of dying at home. Sometimes I've been told, you know, if I die here so-and-so won't be able to sleep here anymore." (Participant 1)

Many respondents attributed patient fear about home death to poor illness understanding, which was associated

Table 2 Identified categories and factors

\begin{tabular}{|c|c|c|}
\hline Category & Definition & Factors \\
\hline Patient Characteristics & $\begin{array}{l}\text { Characteristics related to a patient's background, culture } \\
\text { and character traits, as well as their physical, emotional } \\
\text { or mental state were believed to influence their ability } \\
\text { to die at home. These were described by } 8 / 9 \text { participants. }\end{array}$ & $\begin{array}{l}\text { 1. Patient preferences about place of death } \\
\text { 2. Patient ability to navigate the health care system }\end{array}$ \\
\hline Physical Environment & $\begin{array}{l}\text { A patient's physical environment refers to the location } \\
\text { (home or housing) where they will receive palliative } \\
\text { care. Characteristics pertaining to the physical } \\
\text { environment were believed to influence likelihood of } \\
\text { dying at home. This was described by } 8 / 9 \text { participants. }\end{array}$ & $\begin{array}{l}\text { 1. Environment suitability to accommodate care } \\
\text { 2. Environment stability } \\
\text { 3. Environment safety }\end{array}$ \\
\hline Support Network & $\begin{array}{l}\text { The support network includes any individual who } \\
\text { provided care or assistance to the patient during their } \\
\text { end-of-life care trajectory. Having more support was } \\
\text { equated with a greater likelihood of dying at home. } \\
\text { This was described by } 9 / 9 \text { participants. }\end{array}$ & $\begin{array}{l}\text { 1. Caregiver availability and ability to organize and } \\
\text { coordinate services } \\
\text { 2. Ability to supplement care needs with paid-caregiving } \\
\text { services } \\
\text { 3. Health care provider advocacy for patient needs }\end{array}$ \\
\hline
\end{tabular}


with perceived lower levels of education, language barriers or lack of access to resources.

"If [patients] don't really understand or know what to expect, then they're easily anxious and thus call a lot or go to hospital a lot because they just don't feel comfortable being at home, even though, probably, some aspects of that care can be done quite adequately at home. They just can't deal with that." (Participant 5)

Beyond preference, participants noted that patients required the ability to navigate the health care system, advocate for their health care needs, and assemble a support network. Participants felt that ability to advocate was influenced by a patient's level of education, socioeconomic status, and understanding of their disease trajectory.

"I think the other factor, too, is whether they have somebody advocating for them, so whether they are able to know who to talk to and how to access care... Those who may not have English as the first language or those who are new, don't know who to turn to or who to ask... They don't know if they should be asking or can ask." (Participant 3)

\section{Physical environment}

Factors relating to a patient's physical environment primarily pertained to the suitability, stability and safety of their housing for meeting the needs of patients and caregivers at the end of life. A physical environment was perceived to be suitable to support home death if it was able to accommodate medical equipment and care providers, had access to the basic amenities (e.g. toilet, shower), and was free of hazards (e.g. bedbugs, vermin, mould).

"Sometimes a place is so small or cluttered that you can't put a hospital bed in, or sometimes there are vermin in the dwelling, so [also] physical factors of them not being able to get from one place to the other, not having access to the washroom or a shower." (Participant 1)

Participants felt that patients living without access to reliable or subsidized housing were less likely to die out of an institutionalized setting.

"It's not a widespread problem but for the people who don't have access to housing, it is a big problem" (Participant 9)
Socioeconomic status was perceived to impact these factors, insofar as poverty was associated with less suitable and more precarious housing.

The safety of the home environment also influenced the ability or willingness of providers to care for patients at home. The safety concerns described were typically associated with lower-income situations, and included residing in neighbourhoods with run down buildings, a higher prevalence of mental health issues, and substance abuse issues.

"The other piece is safety. In a lot of these places, can we have things like opioids and controlled substances in a place where if they're alone they need to keep the door unlocked and so who is going to go in and potentially affect them... And then not only myself as a physician but the nursing staff, the safety and a lot of these places unfortunately are deemed unsafe." (Participant 3)

These perceived and actual concerns for personal safety may preclude providers from participating in a patient's after hours care.

"One of the barriers, especially in my area is a lot of those areas are what we call 'no go' zones at night. So, if someone calls after hours or when it's dark, a doctor is not going to go out to see them because it's unsafe." (Participant 5)

Some physicians acknowledged that what constituted a suitable environment for home death can be subjective. When describing patients living in less-suitable environments, some participants appeared to remain objective when evaluating whether a patient might be able to die there.

“In some cases you don't impose your sense of what a good home is, and I may not be willing to live in that kind of environment.... You can't impose- That's not how I would want to live or want to care for someone. I guess we kind of have insight about what we think we might need, so the running water and the shower and room to turn and manoeuvre and all the things that we think the person might need as they get sicker." (Participant 6)

Other physicians suggested that care provider bias about a patient's environment might impact where that patient ultimately dies.

"The sad thing is that it's so integrated into how I think about patients now, almost right from the get 
go. I will have a sense of 'is this going to work or is it not'. And it's not like I would say it's absolutely not going to work if the patient has very limited finances, but it's something that we address much more quickly in the visits. I almost wonder: do we end up steering the patient away from an anticipated home death because we can tell [that it will be difficult to provide end of life care]." (Participant 2)

\section{Support networks}

Support networks included any individuals who provided care or assistance to the patient during their end-of-life care trajectory. Participants highlighted three supportrelated factors that improved a patient's likelihood of dying at home: having family caregivers who are available and able to organize and coordinate services, being able to supplement care needs with paid-caregiving services, and having health care providers who would advocate for patient needs.

"I would say if the family and the patient, but mainly the family, are committed to making it happen at home, and they have enough numbers, possibly more than one committed family member to share the load, then I think it's possible." (Participant 2)

Like the physician above, all respondents highlighted the presence of a caregiver as the most significant factor in achieving a home death, if that is what the patient desires. Physicians noted the key role of caregivers as advocates who organized and coordinated service delivery. Socioeconomic status was perceived to influence caregiver support in two ways. Frist, participants believed that family members who are unable to take time off work for economic reasons are less available to be primary caregivers.

"I think one of the real kind of hidden ways in which socioeconomics comes into play, is when caregivers need to work in order to keep the food on the table and the rent and the hydro paid and all that... So I would love to see a situation where we supported caregivers financially to do caregiving." (Participant 9)

Second, participants felt that caregivers' abilities to advocate were associated with education level, language skills, and familiarity with the health care system. Caregiver emotional factors were also significant; discomfort with home death, or inability to cope with the demands placed on caregivers were frequently cited reasons for transitioning to an institutional setting.
Patients that were perceived to have more financial resources were frequently able to compensate for a lack of informal caregiver support by purchasing private care. This ability to afford private services was consistently described as the primary means by which socioeconomic status impacts home death.

"People with a lower socioeconomic status can't afford to pay for private health because [the home care services organization] cannot provide all the support that's required"... "I have met people who have higher...assets, and they're using those assets to pay for private care, and it makes it possible for them to stay in home because they can, essentially, purchase all of the care that they need that they would get in the palliative care unit." (Participant 1)

Finally, participants perceived health care providers as more likely to take on the responsibility for advocacy when caring for lower SES populations, noting that some clinicians go "above and beyond their duties" to get patients what they need. One explanation provided as to why clinicians were more likely to take on this responsibility for lower SES patients was recognition of the significant difference advocacy could make to patient outcomes in this population.

"In some cases if [patients] have lower socioeconomic statuses and you have people in the health care field that will advocate for you-...It's almost like you may be empathized with a little bit more because [you] have less" (Participant 5)

\section{Strategies to decrease disparities}

Participants identified increasing access to home-based support services, including home care support, personal support workers or other specialized care, as a strategy that might address SES-based disparities in patients' abilities to die at home. As a part of this support, participants felt that it would be beneficial to have a designated provider for assessing, advocating and coordinating services for lower SES patients, instead of considering this to be an extra task, above and beyond one's current duties.

"Maybe even something simple, like making it part of someone's job to do those things, to look at how much money do [the patients] have? How can the system benefit [the patient] the most and advocate for them. But not make it part of [the provider's] job so that they're going above and beyond.... But make it part of someone's job to do that, would probably be the best way [to address discrepancies]" (Participant 5) 
Participants also identified enhancing support for caregivers as a strategy to improve a patient's chances of dying at home. Providing income support for necessities like food and housing was hypothesized to relieve financial and time burden, thus providing caregivers more flexibility to engage as primary caregivers at end of life."-

Somehow providing support for their housing, for food, for being off work, and then more directly, patient care, providing more support for those people who can't afford the extra help, but do need extra help" (Participant 4)

\section{Discussion}

The aim of this study was to identify the key factors perceived to influence likelihood of home death, and describe the mechanisms by which they may interact with SES. Physicians in our study identified multiple factors that were perceived to influence the likelihood of home death. These factors emerged within three main categories: patient characteristics, physical environment, and support networks. Participants indicated that stability within each category was necessary for home death, and that strengths in one could supplement weaknesses in another. For example, a patient's ability to advocate for themselves can bolster their support system by maximizing publicly funded home support. Conversely, some deficits were too large to be compensated for by strength in other categories; for example, if a patient characteristic, such as anxiety, decreases the desire to stay at home, neither an optimal physical environment, nor a robust support system would compensate for this.

Our findings suggest that, while SES is not a primary factor in determining likelihood for home death, it may influence the other main categories of determinants. Higher SES may strengthen support networks, contribute to a more stable home environment, and increase patient comfort with home death. This finding may partially explain why some studies evaluating SES on a population level did not find SES to be a significant predictor of home death [11, 25-31]; our findings suggest that the interaction between SES and other determinants such as social support or preference for place of death is complex, and thus may be obscured in population-level studies. That SES was not identified as an independent key factor of home death in our study may also result from its examination within a home-palliative care setting. In a 2015 systematic review of patients receiving specialized home care, Chen found that receiving specialist care may decrease socioeconomic inequities in access to preferred home death [39]. Similarly, Barclay found that receiving home-hospice palliative care within a continuous care model was able to eliminate disparities in the rates of transfer from home between patients classified as low or high income [40].

Preference for home death [11], and awareness of dying and realistic coping attitudes [41] have been cited as predictors of home death. Our participants indirectly linked SES to preference by way of illness understanding: Patients perceived to have less education, language barriers or lack of access to resources were believed to have poorer illness understanding, and therefore experienced greater discomfort with dying in the home. These findings are consistent with literature that has identified socioeconomic determinants that increase rates of preference for home death, including identifying as non-Hispanic white race [42], greater education level, greater income levels, greater awareness of advanced directives [43], and more supportive living arrangements [44]. These findings can be contrasted with earlier studies, which found that immigrant or direct descendants express a higher preference for home death [45] and a higher likelihood of dying at home [24]. Building on our findings, further research is needed to more completely characterize how socioeconomic factors influence preference for home death.

Finally, support systems comprised of informal caregivers and supplemented with paid-services and dedicated health care providers were felt to improve a patient's likelihood of home death. In Canada, informal caregivers play a significant role in the provision of home care [46-48], and have been found to be predictors of home death [11, 12, 14, 27]. De Conno (1996) found that family support was a greater predictor of home death than either financial or housing conditions [27], reflecting the value of caregiver support described by our participants. Our findings further characterize the link between SES and support networks: higher SES may improve an informal caregiver's capacity to support the patient by decreasing financial pressures, thus allowing more time for caregiving, and by compensating for gaps in informal caregiver support through greater access to paid supports. This is supported by literature. In Canada, $43 \%$ of caregivers indicated that caregiving duties disrupted their normal work routine [49]. Studies have also shown social support networks in lower SES families to have access to fewer resources, thus limiting informal caregiver capacity to provide adequate care [50, 51]. Meanwhile, caregivers with more job flexibility and disposable income are able to provide more care themselves and require fewer home care services [16]. These same caregivers may be better able to advocate for needed services $[17,20]$. Finally, the economic burden that families incur while supporting home death, even within publicly funded health systems, can be significant $[11,21,52,53]$. As Rossi et al. note in their Italian survey of family and caregivers of patients dying of cancer, 
a large proportion of families exhaust almost all of their savings in providing care at the end of life [54], suggesting that families with greater financial resources would face fewer barriers to home death.

\section{Areas of potential intervention}

Strengthening support networks to facilitate home death was a primary focus of our participants, which is supported by the literature. Barclay et al. demonstrated that providing more intensive, continuous home palliative care decreased socioeconomic disparities in incidence of home death [40]. Likewise, Yamagishi et al. (2012) proposed that improving 24-h support at home is required to make home death more accessible [55].

Participants noted that a focus on caregivers, through emotional support, education, and financial assistance could also improve support network robustness, thereby contributing to improved patient state of mind. This complements the findings of Milberg et al. (2014), who found that patients who felt less supported by family had higher degrees of stress, anxiety, and increased worries about personal and financial security [56].

Better education of patients and caregivers, both around illness understanding as well as the structure of the medical system, is also a potential area of intervention. There have been documented difficulties with health literacy and physician communication in lower SES populations [57], so these populations should be especially prioritized.

\section{Limitations}

We recognize that physicians' perspectives may not capture the everyday realities of patients and their families at the end of life. Indeed, physicians may perceive their low SES patients as being more dependent, less responsible and less rational than higher SES patients [58], which may manifest as lack of empathy, compassion and respect for patients resulting in poor utilization of medical services [59]. Monnickendam et al. (2007) also found that social consciousness of physicians was low, and any helping behaviours were largely detached from systemic poverty-related issues [60]. As well, our findings may not be representative of the experiences of health care providers from different disciplines and settings $[18,61,62]$. While our physician participants largely appeared to demonstrate both empathic responses to patients of lower SES, and cogent analyses of the structural impacts of poverty and the systems-level policies that may improve a patient's agency over place of death, it is important to keep in mind potential biases they may bring to this discourse.

Furthermore, we relied on a physician's perception of their patients' socioeconomic statuses, the accuracy of which was not validated. Indeed, participants perceived a wide variation in the socioeconomic make-up of their practices, ranging from $7.5-90 \%$. This may be partly explained by the wide geographic disparities within the city of Toronto, but may also indicate physicians' inaccurate perceptions of the socioeconomic status of their patients. Given that socioeconomic status was perceived to impact place of death, this finding illustrates potential benefit of implementing a formalized poverty screening tool, such as the one described by Brcic et al. [63].The physicians who participated in this study provide care to patients in their homes, which may have informed their perception of their patients' SES. Previous studies have documented that visiting patients in their home can provide additional insight into the gaps patients may face in their care [64].

The generalizability of our findings may be limited due to the fact that our study was based within a publically funded health care system where patients have access to home care and specialized palliative care services, regardless of socioeconomic status. Results may not be as applicable, therefore, in jurisdictions where primary health care is not universally available.

\section{Future research}

While our exploratory data suggest relationships and dependencies among the broad categories that we identified, further research with a larger and more diverse group of informants is necessary to generate a clearer model of home death.

Our respondents did not comment at length on the factors that shape preference for place of death. They did, however, note that emotional stressors do have a significant impact on patient and family comfort with home death. Further study of the determinants of patient preference would assist in addressing any modifiable factors that may arise from socioeconomic disparity.

\section{Conclusions}

Our study provides preliminary insights into the key factors that influence home death, and defines the mechanisms by which they and socioeconomic status interact. These findings were informed by the perceptions of home palliative care physicians in an urban setting in Toronto, Canada. Our participants noted three categories of factors that affect a successful home death: patient characteristics, physical environment, and support networks. While socioeconomic status was not seen by physicians as the primary determinant of a patient's ability to die a home, a higher SES was perceived to interact with these three categories of factors by strengthening support networks, 
optimizing physical home environments, and increasing patient comfort with home death. Possible areas of intervention to increase access to home death focused on better support for patients and families through increased resources and advocacy. Increasing the agency of those who prefer home death in an equitable way is a public policy imperative and should be prioritized by clinicians and policy-makers.

\section{Abbreviations}

FTE: Full time equivalent; SES: Socio-economic status

\section{Funding}

This research was supported by the Temmy Latner Centre for Palliative Care.

\section{Availability of data and materials}

The data used and/or analysed during this study are available from the corresponding author on reasonable request.

\section{Authors' contributions}

JW, AH and AMK contributed to the conceptualization of this study. JW and AMK participated in the development of data collection tools, data analysis, data interpretation, preparation and editing of the manuscript. AMK completed data collection. All authors have read and approved the final manuscript.

\section{Authors' information}

Dr. Joshua Wales is a community palliative care physician at the Temmy Latner Centre for Palliative care. Allison Kurahashi is the Senior Educational Research Coordinator at the Temmy Latner Centre for Palliative Care. Dr. Amna Husain is an Associate Professor in the Department of Family and Community Medicine at the University of Toronto, and Research Lead and palliative care physician at the Temmy Latner Centre for Palliative Care.

\section{Ethics approval and consent to participate}

This study protocol was approved by the Mount Sinai Hospital Research Ethics board (Study ID 15-0303-E). Informed consent was obtained from all participants prior to participation.

\section{Consent for publication}

Not applicable

\section{Competing interests}

The authors declare that they have no competing interests.

\section{Publisher's Note}

Springer Nature remains neutral with regard to jurisdictional claims in published maps and institutional affiliations.

Received: 9 May 2017 Accepted: 12 June 2018

Published online: 20 June 2018

\section{References}

1. Agar M, Currow DC, Shelby-James TM, et al. Preference for place of care and place of death in palliative care: are these different questions? Palliat Med. 2008;22(7):787-95. https://doi.org/10.1177/0269216308092287.

2. Gomes B, Higginson IJ, Calanzani N, et al. Preferences for place of death if faced with advanced cancer: a population survey in England, Flanders, Germany, Italy, the Netherlands, Portugal and Spain. Ann Oncol. 2012;23(8): 2006-15. https://doi.org/10.1093/annonc/mdr602.

3. Higginson IJ, Sen-Gupta GJ. Place of care in advanced cancer: a qualitative systematic literature review of patient preferences. J Palliat Med. 2000;3(3): 287-300. https://doi.org/10.1089/jpm.2000.3.287.

4. Zimmer JG, Groth-Juncker A, McCusker J. Effects of a physician-led home care team on terminal care. J Am Geriatr Soc. 1984;32(4):288-92. https://doi. org/10.1111/j.1532-5415.1984.tb02023.x.

5. Burge F, Lawson B, Johnston $G$. Trends in the place of death of cancer patients, 1992-1997. CMAJ. 2003:168(3):265-70. [published Online First: 2003/02/05]
6. Gomes B, Higginson IJ. Factors influencing death at home in terminally ill patients with cancer: systematic review. Br Med J. 2006;332(February):515-8. https://doi.org/10.1136/bmj.38740.614954.55.

7. Weitzen S, Teno JM, Fennell M, et al. Factors associated with site of death: a national study of where people die. Med Care. 2003;41(2):323-35. https:// doi.org/10.1097/01.MLR.0000044913.37084.27.

8. Bruera E, Sweeney C, Russell N, et al. Place of death of Houston area residents with cancer over a two-year period. J Pain Symptom Manag. 2003; 26(1):637-43. [published Online First: 2003/07/10]

9. Cárdenas-Turanzas M, Carrillo MT, Tovalín-Ahumada H, et al. Factors associated with place of death of cancer patients in the Mexico City metropolitan area. Support Care Cancer. 2007;15(3):243-9. https://doi.org/10. 1007/s00520-006-0152-4

10. Gallo WT, Baker MJ, Bradley EH. Factors associated with home versus institutional death among cancer patients in Connecticut. J Am Geriatr Soc. 2001;49(6):771-7. https://doi.org/10.1046/j.1532-5415.2001.49154.x

11. Guerriere DN, Husain A, Marshall D, et al. Predictors of place of death for those in receipt of home-based palliative Care Services in Ontario, Canada. J Palliat Care. 2015;31(2):76-88.

12. Houttekier D, Cohen J, Bilsen J, et al. Determinants of the place of death in the Brussels metropolitan region. J Pain Symptom Manag. 2009:37(6):9961005. https://doi.org/10.1016/j.jpainsymman.2008.05.014.

13. Karlsen $\mathrm{S}$, Addington-Hall J. How do cancer patients who die at home differ from those who die elsewhere? Palliat Med. 1998;12(4):279-86. https://doi. org/10.1191/026921698673427657.

14. Aoun S, Kristjanson L, Currow D, et al. Terminally-ill people living alone without a caregiver: an Australian national scoping study of palliative care needs. Palliat Med. 2007;21(1):29-34. https://doi.org/10.1177/ 0269216306073198. [published Online First: 2006/12/16]

15. Barbera L, Sussman J, Viola R, et al. Factors associated with end-of-life health service use in patients dying of Cancer. Healthc Policy. 2010;5(3):e125-43. https://doi.org/10.12927/hcpol.2013.21644.

16. Cai J, Guerriere DN, Zhao H, et al. Socioeconomic differences in and predictors of home-based palliative care health service use in Ontario, Canada. Int J Environ Res Public Health. 2017;14(7) https://doi.org/10.3390/ ijerph14070802. [published Online First: 2017/07/19]

17. Howell D, Abernathy T, Cockerill R, et al. Predictors of home care expenditures and death at home for Cancer patients in an integrated comprehensive palliative home care pilot program. Healthc Policy. 2011;6(3): 73-92. https://doi.org/10.12927/hcpol.2011.22179.

18. Burge Fl, Lawson B, Johnston $\mathrm{G}$. Home visits by family physicians during the end-of-life: does patient income or residence play a role? BMC Palliat Care. 2005;4(1):1. https://doi.org/10.1186/1472-684X-4-1.

19. Decker SL, Higginson IJ. A tale of two cities: factors affecting place of cancer death in London and New York. Eur J Pub Health. 2007:17(3):285-90. https://doi.org/10.1093/eurpub/ckl243.

20. Grande G, Addington-Hall J, Todd C. Place of death and access to home care services: are certain patient groups at a disadvantage? Soc Sci Med. 1998:47(5):565-79. https://doi.org/10.1016/S0277-9536(98)00115-4.

21. Higginson IJ, Costantini M. Dying with cancer, living well with advanced cancer. Eur J Cancer. 2008;44(10):1414-24. https://doi.org/10.1016/j.ejca.2008. 02.024 .

22. Higginson IJ, Jarman B, Astin P, et al. Do social factors affect where patients die: an analysis of 10 years of cancer deaths in England. J Public Health Med. 1999;21(1):22-8. https://doi.org/10.1093/pubmed/21.1.22.

23. McCusker J. Where cancer patients die: an epidemiologic study. Public Health Rep (Washington, DC : 1974). 1983;98(2):170-6.

24. Motiwala SS, Croxford R, Guerriere DN, et al. Predictors of place of death for seniors in Ontario: a population-based cohort analysis. Can J Aging. 2006; 25(4):363-71. https://doi.org/10.1353/cja.2007.0019.

25. Fukui S, Kawagoe H, Masako S, et al. Determinants of the place of death among terminally ill cancer patients under home hospice care in Japan. Palliat Med. 2003;17(5):445-53. https://doi.org/10.1191/0269216303pm782oa. [published Online First: 2003/07/29]

26. Fukui S, Fukui N, Kawagoe H. Predictors of place of death for Japanese patients with advanced-stage malignant disease in home care settings: a nationwide survey. Cancer. 2004;101(2):421-9. https://doi.org/10.1002/cncr. 20383. [published Online First: 2004/07/09]

27. De Conno F, Caraceni A, Groff $L$, et al. Effect of home care on the place of death of advanced cancer patients. Eur J Cancer (Oxford, England : 1990). 1996;32(7):1142-7. 
28. Cantwell $P$, Turco $S$, Brenneis $C$, et al. Predictors of home death in palliative care cancer patients. J Palliat Care. 2000;16(1):23-8. [published Online First: 2000/05/10]

29. Fukui S, Fujita J, Tsujimura M, et al. Predictors of home death of home palliative cancer care patients: a cross-sectional nationwide survey. Int J Nurs Stud. 2011;48(11):1393-400. https://doi.org/10.1016/j.jjnurstu.2011.05. 001. [published Online First: 2011/05/31]

30. Masucci L, Guerriere DN, Cheng R, et al. Determinants of place of death for recipients of home-based palliative care. J Palliat Care. 2010;26(4):279-86. [published Online First: 2011/01/28]

31. Alonso-Babarro A, Bruera E, Varela-Cerdeira $M$, et al. Can this patient be discharged home? Factors associated with at-home death among patients with cancer. J Clin Oncol. 2011;29(9):1159-67. https://doi.org/10.1200/JCO. 2010.31.6752. [published Online First: 2011/02/24]

32. Sandelowski M. What's in a name? Qualitative description revisited. Res Nurs Health. 2009;23(4):334-40. https://doi.org/10.1002/nur.20362.

33. Elo S, Kyngas $\mathrm{H}$. The qualitative content analysis process. J Adv Nurs. 2008; 62(1):107-15. https://doi.org/10.1111/j.1365-2648.2007.04569.x. [published Online First: 2008/03/21]

34. Tong A, Sainsbury P, Craig J. Consolidated criteria for reporting qualitative research (COREQ): a 32-item checklist for interviews and focus groups. Int J Qual Health Care. 2007;19(6):349-57. https://doi.org/10.1093/intqhd/ mzm042. [published Online First: 2007/09/18]

35. Social Policy Analysis \& Research. Profile of low income in the city of Toronto. Toronto Demographics. Toronto: City of Toronto; 2011.

36. Hennink MM, Kaiser BN, Marconi VC. Code saturation versus meaning saturation: how many interviews are enough? Qual Health Res. 2017;27(4): 591-608. https://doi.org/10.1177/1049732316665344. [published Online First: 2016/09/28]

37. Kerr C, Nixon A, Wild D. Assessing and demonstrating data saturation in qualitative inquiry supporting patient-reported outcomes research. Expert Rev Pharmacoecon Outcomes Res. 2010;10(3):269-81. https://doi.org/10. 1586/erp.10.30. [published Online First: 2010/06/16]

38. M. Morse J, Barrett M, Mayan M, et al. Verification strategies for establishing reliability and validity in qualitative research. Int J Qual Methods 2002;1(2): 13-22. doi: https://doi.org/10.1177/160940690200100202.

39. Chen $\mathrm{H}$, Nicolson DJ, Macleod U, et al. Does the use of specialist palliative care services modify the effect of socioeconomic status on place of death? A systematic review. Palliat Med. 2015; https://doi.org/10.1177/ 0269216315602590.

40. Barclay JS, Kuchibhatla M, Tulsky JA, et al. Association of hospice patients' income and care level with place of death. JAMA Intern Med. 2013;173(6): 450-6. https://doi.org/10.1001/jamainternmed.2013.2773. [published Online First: 2013/02/20]

41. Hinton J. Which patients with terminal cancer are admitted from home care? Palliat Med. 1994;8(3):197-210. https://doi.org/10.1177/ 026921639400800303. [published Online First: 1994/01/01]

42. Barnato $A E$, Anthony $D L$, Skinner J, et al. Racial and ethnic differences in preferences for end-of-life treatment. J Gen Intern Med. 2009;24(6):695-701. https://doi.org/10.1007/s1 1606-009-0952-6. [published Online First: 2009/04/24]

43. Foreman LM, Hunt RW, Luke CG, et al. Factors predictive of preferred place of death in the general population of South Australia. Palliat Med. 2006; 20(4):447-53. https://doi.org/10.1191/0269216306pm1149oa. [published Online First: 2006/08/01]

44. lecovich E, Carmel S, Bachner YG. Where they want to die: correlates of elderly persons' preferences for death site. Soc Work Public Health. 2009; 24(6):527-42. https://doi.org/10.1080/19371910802679341. [published Online First: 2009/10/13]

45. Schou-Andersen $M$, Ullersted MP, Jensen $A B$, et al. Factors associated with preference for dying at home among terminally ill patients with cancer. Scand J Caring Sci. 2016;30(3):466-76. https://doi.org/10.1111/scs.12265. [published Online First: 2015/09/24]

46. Donner G, Fooks C, McReynolds J, et al. Bringing care home. Toronto: Ministry of Health and Long Germ Care; 2015.

47. Sinha M, Bleakney A. Spotlight on Canadians: results from the General Social Survey. Receiving care at home. Ottawa: Statistics Canada; 2014

48. Um S-g, Lightman N. Ensuring healthy aging for all: home care access for diverse senior populations in the GTA. Toronto: Wellesley Institute; 2016

49. Sinha M. Spotlight on Canadians: results from the General Social Survey. Portrait of caregivers, 2012. Ottawa: Statistics Canada; 2013.
50. Lewis JM, DiGiacomo M, Currow DC, et al. Social capital in a lower socioeconomic palliative care population: a qualitative investigation of individual, community and civic networks and relations. BMC Palliat Care. 2014;13(1):30. https://doi.org/10.1186/1472-684X-13-30.

51. The Change Foundation. A profile of family caregivers in Ontario. Toronto: The Change Foundation; 2016.

52. Chochinov HM, Janson K. Dying to pay: the cost of end of life care. J Palliat Care. 1998;14(4):5-15.

53. Hanratty B, Jacoby A, Whitehead M. Socioeconomic differences in service use, payment and receipt of illness-related benefits in the last year of life: findings from the British household panel survey. Palliat Med. 2008;22(3): 248-55. https://doi.org/10.1177/0269216307087140.

54. Rossi PG, Beccaro M, Miccinesi $G$, et al. Dying of cancer in Italy: impact on family and caregiver. The Italian survey of dying of Cancer. J Epidemiol Community Health. 2007;61(6):547-54. https://doi.org/10.1136/jech.2005. 045138.

55. Yamagishi A, Morita T, Miyashita M, et al. Preferred place of care and place of death of the general public and cancer patients in Japan. Support Care Cancer. 2012;20(10):2575-82. https://doi.org/10.1007/s00520-011-1373-8.

56. Milberg A, Wåhlberg R, Krevers B. Patients ' sense of support within the family in the palliative care context: what are the influencing factors? Psychooncology. 2014;23(12):1340-9. https://doi.org/10.1002/pon.3564.

57. Lewis JM, DiGiacomo M, Currow DC, et al. Dying in the margins: understanding palliative care and socioeconomic deprivation in the developed world. J Pain Symptom Manag. 2011;42(1):105-18. https://doi. org/10.1016/j.jpainsymman.2010.10.265.

58. van Ryn M, Burke J. The effect of patient race and socio-economic status on physicians' perceptions of patients. Soc Sci Med. 2000;50(6):813-28. https:// doi.org/10.1016/S0277-9536(99)00338-X.

59. Stewart M, Reutter L, Makwarimba E, et al. Determinants of health-service use by low-income people. Can J Nurs Res. 2005;37(3):104-31. [published Online First: 2005/11/05]

60. Monnickendam M, Monnickendam SM, Katz C, et al. Health care for the poor-an exploration of primary-care physicians' perceptions of poor patients and of their helping behaviors. Soc Sci Med. 2007;64(7):1463-74. https://doi.org/10.1016/j.socscimed.2006.11.033.

61. Dumont $S$, Jacobs $P$, Turcotte $V$, et al. Palliative care costs in Canada: descriptive comparison of studies of urban and rural patients near end of life. Palliat Med. 2015;29(10):908-17. https://doi.org/10.1177/ 0269216315583620. [published Online First: 2015/06/05]

62. Goodridge D, Lawson J, Rennie D, et al. Rural/urban differences in health care utilization and place of death for persons with respiratory illness in the last year of life. Rural Remote Health. 2010;10(2):1349. [published Online First: 2010/05/05]

63. Brcic V, Eberdt C, Kaczorowski J. Development of a tool to identify poverty in a family practice setting: a pilot study. Int J Family Med. 2011;2011: 812182. https://doi.org/10.1155/2011/812182. [published Online First: 2012/ 02/09]

64. Hervada-Page M, Fayock KS, Sifri R, et al. The home visit experience: a medical student's perspective. Care Manag J. 2007:8(4):206-10. [published Online First: 2008/02/02]

\section{Ready to submit your research? Choose BMC and benefit from:}

- fast, convenient online submission

- thorough peer review by experienced researchers in your field

- rapid publication on acceptance

- support for research data, including large and complex data types

- gold Open Access which fosters wider collaboration and increased citations

- maximum visibility for your research: over $100 \mathrm{M}$ website views per year

At BMC, research is always in progress.

Learn more biomedcentral.com/submissions 